\title{
Nonlinear Dynamics of a Planar Hinged-Supported Beam with One End Lumped Mass and Longitudinal Elastic Support
}

\author{
Lukasz Kloda ${ }^{1,2^{*}}$, Stefano Lenci ${ }^{1}$, and Jerzy Warminski ${ }^{2}$ \\ ${ }^{1}$ Department of Civil and Buildings Engineering, and Architecture, Polytechnic University of Marche, Ancona, Italy \\ ${ }^{2}$ Department of Applied Mechanics, Lublin University of Technology, Lublin, Poland
}

\begin{abstract}
Nonlinear forced-damped oscillations of a non-slender hinged simply supported beam with mass and spring attached to one end are investigated by mean of a finite element method. The frequency response curves are constructed numerically and the variability of hardening/softening behaviour of frequency response curves due to the lumped mass and axial linear spring stiffness is investigated. Resonant and sub resonant motion of beam midpoint as well as jumps between solution branches are highlighted.
\end{abstract}

\section{Introduction}

In mechanical systems undergoing incidental or operational excitation, a special attention must be paid to nonlinear dynamic phenomena which must be well understood by mechanical and structural engineers. Nonlinear dynamics effects may arise especially in the vicinity of natural frequencies. Currently at the initial designing stage a real structure can be adequately modelled numerically e.g. by finite element method and then examined to understand and optimize the way it works, without building advanced laboratory test devices or sophisticated analytical algorithms. Moreover, such numerical analysis is available to cover predictable global phenomena in a wide range as well as unexpected local effects, see $[1,2]$. The main disadvantages of this approach is studying only specific cases and needing huge computational time for very complex systems. However, a development of computing machines increases significantly possibilities of numerical models enabling to detect special effects which are difficult to measure. It causes that unreachable things for today can be common in the near future.

Recently a few papers have been published on coupled transversal and longitudinal oscillations of beams with different boundary conditions. Application of passive control by arbitrary assumed end mass and varying one end stiffness of a spring kinematically excited in the longitudinal direction is presented in [3]. In [4] authors focused on parametrically excited beamaxial spring-lumped mass system. However, the ratio of the beam mass and tip mass was assumed as a very small number thus the study was limited to a particular case.
In this paper we are focused on numerical computations of a planar beam-spring system, wherein the effect of the additional end mass is investigated. Namely, a hinged-simply supported beam with a tip mass and a linear spring axially restraining one end of the beam is studied (Fig. 1). The added end mass can be understood for small/medium values as an effective mass of the spring; passive control/balancing frequency response curves for moderate values; or due to other reasons as a huge tip mass which have to be carried out as a part of the global system. Regardless of the mass origin, it always appears in real applications which is the motivation for research. Besides, frequency response curves hysteresis and an associated jump phenomenon will be addressed in this work.

\section{Finite element model}

The finite element model of the structure consists of 100 straight equal length beam elements of B31-type with linear geometric order; linear spring of stiffens $k_{s}$; tip mass $M_{t}$ subjected to one end which undergoes only translatory inertia in the longitudinal direction (mass rotation is neglected). Beam characteristic parameters are collected in Table 1. The corresponding boundary conditions are:

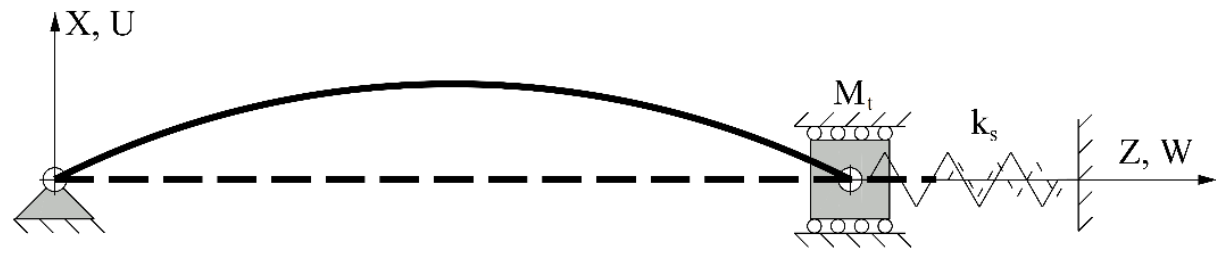

Fig. 1. The beam-spring-tip mass system. Thick curve - deformed beam, thick dashed line - rest configuration.

* Corresponding author: 1.kloda@pollub.pl 


$$
\begin{gathered}
U(0, T)=U(L, T)=0, \\
M(0, T)=M(L, T)=0, \\
W(0, T)=0,
\end{gathered}
$$

where Eq. (2) corresponds to vanishing of bending moments at beam ends. The right end side of the beam is ruled by the balance equation in the axial direction (see Fig. 2):

$$
N(L, T) \cos \varphi+V(L, T) \sin \varphi+k_{s} W(L, T)-
$$$$
M_{t} \ddot{W}(L, T)=0 \text {. }
$$

where: $N$ - normal force, $V$ - shear force, $k_{s}$ - spring stiffness, $M_{t}$ - tip mas, $\varphi$ - slope angle, $L$ - beam length in initial unrestrained rest configuration. A dot means derivative with respect to time $T$.

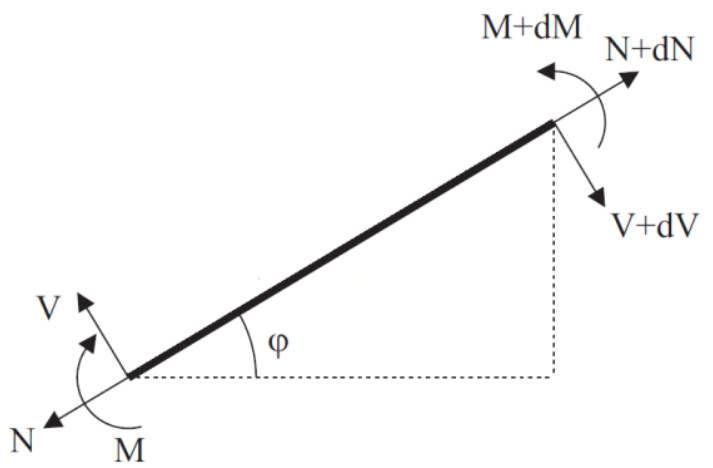

\begin{tabular}{|c|c|c|}
\hline \multicolumn{2}{|c|}{ Initial length $[L]$} & $500 \mathrm{~mm}$ \\
\hline \multicolumn{2}{|c|}{ Cross section $[A]$} & $50 \mathrm{~mm} \times 50 \mathrm{~mm}$ \\
\hline \multicolumn{2}{|c|}{ Density $[\rho]$} & $7850 \mathrm{~kg} / \mathrm{m}^{3}$ \\
\hline \multicolumn{2}{|c|}{ Young modulus $[E]$} & $210 \mathrm{Gpa}$ \\
\hline \multicolumn{2}{|c|}{ Poisson's ratio $[v]$} & 0.3 \\
\hline \multicolumn{2}{|c|}{ Beams shear factor $[\mathrm{Sk}]$} & 0.85 \\
\hline \multicolumn{2}{|c|}{ 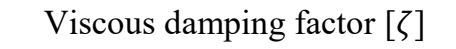 } & $6 \%$ \\
\hline \multicolumn{2}{|c|}{ Tip mass $\left[\mathrm{M}_{\mathrm{t}}\right]$} & $\begin{array}{l}0 \leq M_{t}<\infty \\
(0,1 \mathrm{~kg}, 2 \mathrm{~kg}\end{array}$ \\
\hline \multirow{3}{*}{ 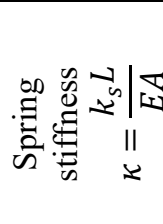 } & $\begin{array}{l}\text { simply supported } \\
\text { end }\end{array}$ & $\kappa=0$ \\
\hline & $\begin{array}{l}\text { free to move with } \\
\text { end spring }\end{array}$ & $\begin{array}{c}0<\kappa<\infty \\
(\kappa=1)\end{array}$ \\
\hline & hinged & $\kappa=\infty$ \\
\hline \multicolumn{2}{|c|}{ Excitation amplitude $\left[p_{v}\right]$} & $40.7992 \mathrm{kN}$ \\
\hline
\end{tabular}

Fig. 2. Deformed beam element - coplanar forces.

Table 1. Beam dimensions and system mechanical properties [5].
It is worth to remark that for zero spring stiffness the system with tip mass is free to move, for $k_{s}$ equals to infinity the joint becomes independent of the end mass, restrained (typically hinged). Between those two limit values of stiffness $\left(0<k_{s}<\infty\right)$ the system turns out to be free to move-spring-tip mass boundary value problem in time domain.

End mas and spring stiffness do not influence the transversal linear natural bending modes, but they affect the longitudinal natural frequencies and corresponding linear modes. However, for larger deformations those modes are coupled and then due to nonlinear dynamics of the beam motion is much more complex.

To study forced oscillations, in simulations, the beam midpoint has been excited in vertical direction (in $X$-direction, see Fig. 1) by a periodic force of amplitude $p_{v}$ which corresponds to $1 \mathrm{~mm}$ static deflection of hinged simply supported beam and circular frequency $\alpha \omega_{0}$ :

$$
P(T)=p_{v} \cos \left(\alpha \omega_{0} T\right),
$$

where: $\omega_{0}$ is natural frequency of the first bending mode; $\alpha$ is multiplicative detuning coefficient.

In order to obtain the frequency response curves, $\alpha$ has been varied in the range from 0.8 to 1.125 , around the first natural frequency $\omega_{0}(461.47 \mathrm{~Hz}, 2899.50 \mathrm{rad} / \mathrm{s})$, step by step forward as well as backward, by disregarding the transient behaviour and keeping only the steady state solution. More precisely, the amplitude of steady state transversal oscillations of the node $Z=L / 2$ has been recorded with sampling at each $1 / 40$ of the period of frequency excitation $\alpha \omega_{0}$. The motion in the longitudinal direction, which in general has double frequency of lateral oscillations, has been recorded, too.

\section{Results}

At the beginning the time histories of the recorded point have been analysed only for selected excitation frequencies, but even those preliminary simulations have brought some phenomena which need deeper investigations. One of them is the effect of the tip mass on the trajectory of the beam midpoint, which undergoes (as expected) coupled motion because axial inertia (of both beam and tip mass), as confirmed by the shifts the intersection of symmetry axis $(U=0)$. In Fig. 3a we show that for small tip mass $M_{t}=2 \mathrm{~kg}$ trajectories are moved toward right of about $0.00132 \mathrm{~mm}$ for $\alpha=0.8$ and $0.00812 \mathrm{~mm}$ for $\alpha=1.1$. Fig. $3 \mathrm{~b}$, on the other hand, presents huge oscillations $(21.45 \mathrm{~mm})$ for extremely big tip mass $(1000 \mathrm{~kg})$, which cause orbits shifts toward left of about $0.265 \mathrm{~mm}$ and $0.00775-0.0021 \mathrm{~mm}$ for $\alpha=0.8$ and $\alpha=1.1$, respectively. Furthermore, for huge mass the orbit is bend right, differently to what happens with small or no tip mass. This proves that the end mass can significantly change dynamics of the beam, and it is necessary to carry out a deeper analysis. 


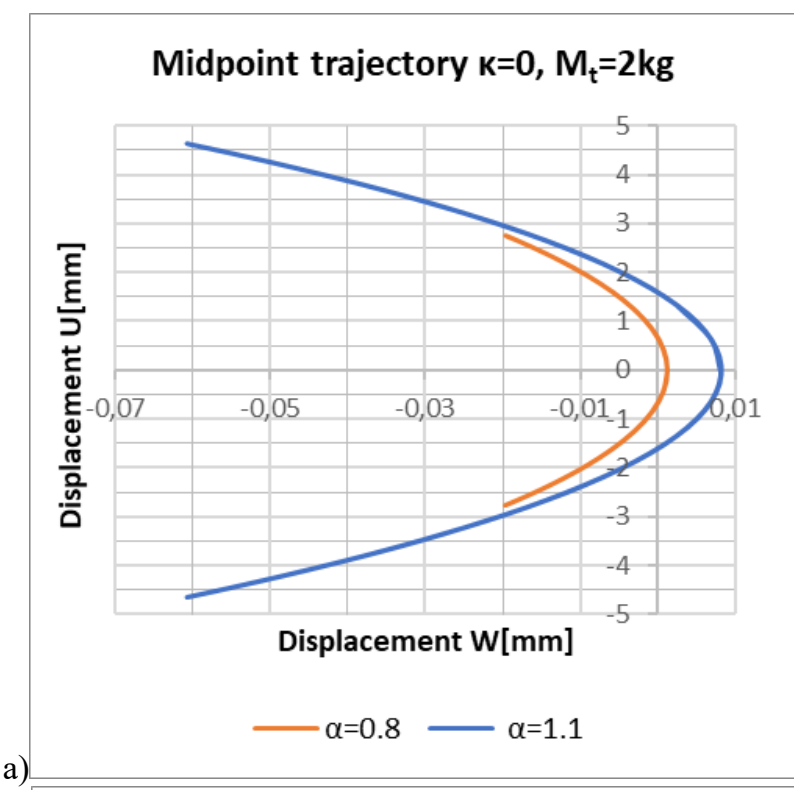

\section{Midpoint trajectory $\mathrm{k}=1, \mathrm{M}_{\mathrm{t}}=1000 \mathrm{~kg}$}

b)

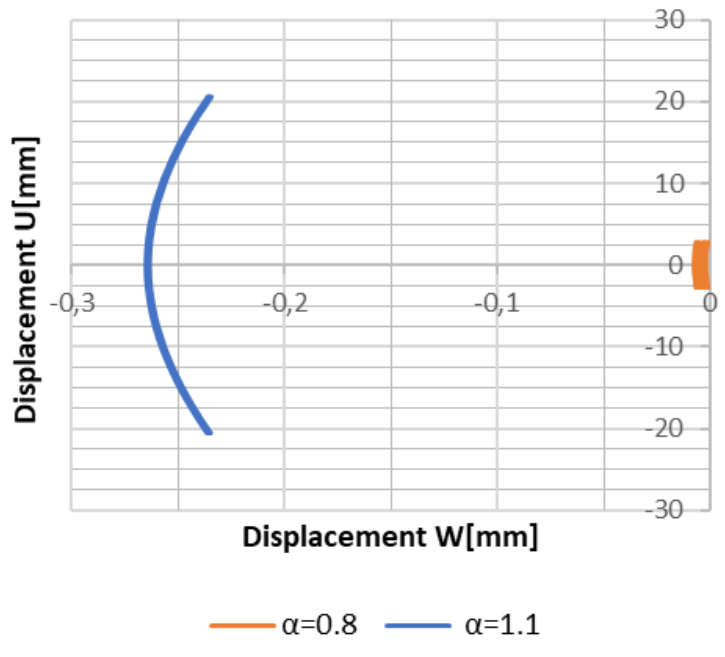

Midpoint trajectory $\mathrm{k}=1, \mathrm{M}_{\mathrm{t}}=1000 \mathrm{~kg}$

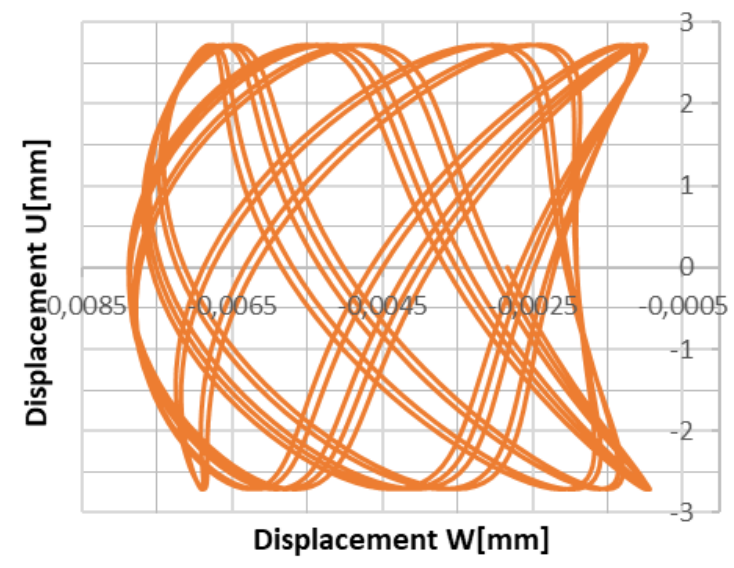

c)

Fig. 3. Orbits of beam mid point for two diffrent frequencies of excitation $\omega_{n}=0.8$ and $\omega_{n}=1.1$ for spring stiffnesses: (a) $\kappa=0, M_{t}=1 \mathrm{~kg},(\mathrm{~b}, \mathrm{c}) \kappa=1, M_{t}=1000 \mathrm{~kg}$.
For system parameters $\kappa=1, M_{t}=1000 \mathrm{~kg}$ and $\alpha=0.8$, a multifrequency response has been detected (see Fig. 3c, which is a zoom of Fig. 3b). Motion of the recorded node is composed of two frequencies $\omega=230.47 \mathrm{~Hz}(1448.09 \mathrm{rad} / \mathrm{s})$ and $\omega=738.28 \mathrm{~Hz}$ $(4638.75 \mathrm{rad} / \mathrm{s})$, which correspond to the frequency of the first longitudinal mode $\omega=230.26 \mathrm{~Hz}(1446.77$ $\mathrm{rad} / \mathrm{s})$ and frequency of the excitation $\omega=0.8 \omega_{0}=738.35 \mathrm{~Hz}(4639.19 \mathrm{rad} / \mathrm{s})$, respectively. This is another relevant dynamical property of the considered system, which could be better analysed in the future.

The numerically obtained Frequency Response Curves (FRC) are given in Fig. 4. As already presented in [2] for the beam with no additional mass, the FRC in the absence of the end spring is softening, while as the end spring stiffness increases the system becomes hardening up to the limit point of restrained end $(\kappa=\infty)$. Additional small end mass causes mollification of characteristics, e.g. for simply supported beam with $M_{t}=2 \mathrm{~kg}(20,38 \%$ of beam mass) the behaviour is significantly more softening, while for $\kappa=1, M_{t}=1 \mathrm{~kg}(10,19 \%)$ it has the same tendency with negligible differences. The interesting thing is that a beam with huge mass $M_{t}=1000 \mathrm{~kg}$ has hardening nature. Besides, the additional mass affects the simply supported beam more than restrained by the spring. The examined cases give a motivation to deeper analytical works in the further investigations.

The well-known jump phenomenon has been observed, both for the softening behavior $\left(\kappa=0, M_{t}=\right.$ $2 \mathrm{~kg}$ and $\kappa=0, M_{t}=0 \mathrm{~kg}$ ) as well as for the hardening one (in this case the upper jump points cannot be seen in Fig. 4 due to the limited range of excitation frequency, but they can be easily foreseen).

\section{Frequency response curves}

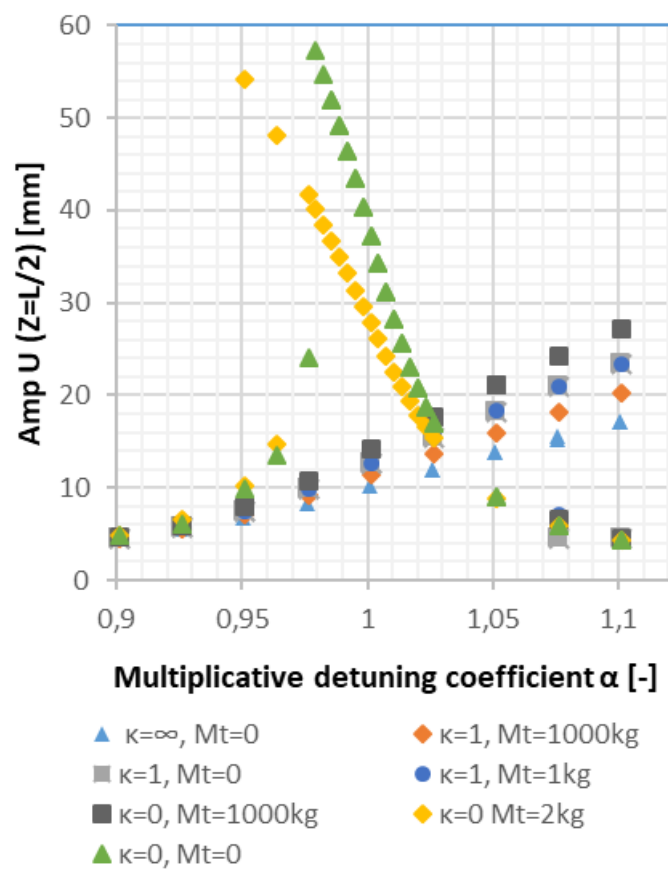

Fig. 4. Frequency response curves - selected system parameters 


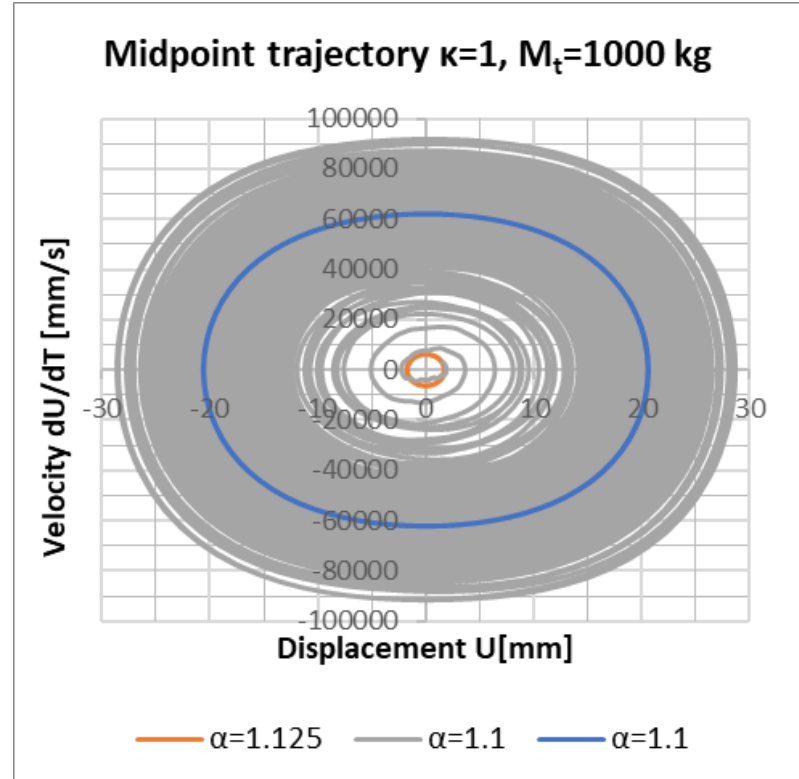

Fig. 5. Trajectories across the jump between large and small amplitude solutions.

This jump phenomenon is now better illustrated. Figure 5 presents, in the phase space $(U, \dot{U})$, the trajectories of the midpoint across the jump phenomenon. The internal small ellipse (orange color) is a picture of the steady state small amplitude oscillation for $\alpha=1.125$. Through a gradual decrement of the excitation frequency (from $\alpha=1.125$ to $\alpha=1.1$ ) the solution has been smoothly perturbed, then the trajectory went out from original steady state up to the other large amplitude oscillation, represented by external ellipse (blue color). The widest area (grey color) is the long transient needed for the stabilization of the system on the new solution. For higher damping factor motion stabilizes much faster in the aftermath of the jump, although it changes the frequency response curve.

\section{Conclusions}

The paper shows some aspects of the nonlinear dynamics of selected cases of a simply supported beam-springlumped mass system, modelled by the finite element method (the commercial software Abacus_CAE® is used). The influence of the tip mass on nonlinear forceddamped oscillations of the beam has been examined. Two scenarios have been considered, with (a) relatively small ratio tip mass to beam mass $10-20 \%(1-2 \mathrm{~kg})$ and for (b) a huge added mass $10000 \%$ (1000 kg). It enables to control the frequency response curves by mean of the added tip mass, which can be useful in practical applications. However, changes are more essential for small spring stiffnesses. The jump between solutions and coexisting trajectory stabilization have been observed numerically.

\section{Acknowledgement}

This work is part of the collaboration between Polytechnic University of Marche and Lublin University of Technology, which is aimed at developing a Joined Doctoral Programme.

\section{References}

1. L. Kloda, S. Lenci, J. Warminski, Nonlinear dynamics of a planar beam-spring system: analytical and numerical approaches, J. Nonlinear Dyn. PDF avaliable online at https://doi.org/10.1007/s11071018-4452-2 (2018) 1-18.

2. L. Kloda, S. Lenci, J. Warminski, Nonlinear dynamics of a planar hinged-supported beam with one end spring system, MATEC Web of Conferences 148 (2018) 06004.

3. A. Shibata, S. Ohishi, H. Yabuno, Passive method for controlling the nonlinear characteristics in a parametrically excited hinged-hinged beam by the addition of a linear spring, J. of Sound Vib. 350 (2015) 111-122.

4. A. Aurami, H. Yabuno, Cubic-quintic nonlinear parametric resonance of a simply supported beam, J. Nonlinear Dyn. 90 (2017) 549-560.

5. Abaqus 6.14 Documentation available at http://dsk.ippt.pan.pl/docs/abaqus/v6.13/index.html (2013). 